\title{
Cultura emprendedora y sustentable de los egresados en carreras empresariales de la Universidad Técnica de Machala
}

\author{
Entrepreneurial and sustainable culture of business graduates from the \\ Technical University of Machala
}

\section{Cultura empreendedora e sustentável de graduados em negócios pela Universidade Técnica de Machala}

\author{
《Jonathan Ruiz Carrillo \\ jruiz@utmachala.edu.ec \\ ORCID: 0000-0002-9183-0004
}

Universidad Nacional Mayor San Marcos, Perú
《Lady León Serrano

llady@utmachala.edu.ec

ORCID: 0000-0001-5472-140X

Universidad del Zulia, Venezuela

Recibido: 12-03-2018 / Revisado: 19-03-2018 / Aceptado: 27-03-2018 / Publicado: 10-09-2018

RESUMEN

En la investigación se analizan las características de comportamiento que definen la cultura emprendedora que han desarrollado los egresados de carreras empresariales, y la sustentabilidad de sus ideas. La investigación es de naturaleza cualitativa con un enfoque cuantitativo en la escala de medición. El estudio se centró en la oferta académica de la Universidad Técnica de Machala, aplicando un test a 804 estudiantes, el mismo que permite estimar los hábitos que ellos tienen a nivel profesional. Estos elementos son los indicadores, cuyos resultados muestran que en la Carrera de Comercio Internacional se maneja una mejor inducción a los alumnos para que formen una cultura emprendedora y sustentable. La fortaleza de estos estudiantes se localiza en sus necesidades de logro. Por tanto, la sustentabilidad de las ideas de estos egresados dependería de la inducción recibida a través de proyectos e investigaciones que ellos desarrollen o en las que participen durante su formación profesional en la universidad referida

Palabras clave: Cultura emprendedora; características de comportamiento, hábitos emprendedores

\begin{abstract}
The research analyzes the behavioral characteristics that define the entrepreneurial culture that graduates of business careers have developed, and the sustainability of their ideas. The research is qualitative in nature with a quantitative focus on the scale of measurement. The study focused on the academic offer of the Technical University of Machala, applying a test to 804 students, the same one that allows estimating the habits they have at a professional level. These elements are the indicators, the results of which show that in the International Trade Career a better induction is handled to the students so that they form an entrepreneurial and sustainable culture. The strength of these students is located in their achievement needs. Therefore, the sustainability of the ideas of these graduates would depend on the induction received through projects and investigations that they develop or in which they participate during their professional training at the referred university.
\end{abstract}

Key words: Entrepreneurial culture; behavioral characteristics, entrepreneurial habits
RESUMO

A pesquisa analisa as características comportamentais que definem a cultura empreendedora que os graduados em carreira de negócios desenvolveram e a sustentabilidade de suas ideias. A pesquisa é de natureza qualitativa com foco quantitativo na escala de medição. O estudo incidiu sobre a oferta académica da Universidade Técnica de Machala, aplicando um teste a 804 alunos, o mesmo que permite estimar os hábitos que possuem a nível profissional. Esses elementos são os indicadores, cujos resultados mostram que na Carreira em Comércio Internacional se dá uma melhor indução aos alunos para que formem uma cultura empreendedora e sustentável. A força desses alunos está localizada em suas necessidades de realização. Portanto, a sustentabilidade das ideias desses egressos dependeria da indução recebida por meio de projetos e pesquisas que desenvolvam ou das quais participem durante sua formação profissional na referida universidade.

Palavras-chave: Cultura empreendedora; características comportamentais, hábitos empreendedores 


\section{INTRODUCCIÓN}

$\mathrm{E}$ I espíritu emprendedor suele entenderse como algo nato de una persona que llega a tener éxito en alguna clase de negocio, no obstante, este concepto puede ser errado al no considerar que el sentido de innovar o abrir nuevas opciones empresariales es algo que cualquier persona puede aprender o perfeccionar. Olvidarse de esperar por un sueldo o salario, o confiar en que nuestras inversiones tomarán siempre un buen rumbo dependiendo de la experiencia empresarial de quienes las operen, dado que esto último es adquirible a nivel cultural y educativo, de forma individual, en sociedad o en familia. Todo esto se ha de desarrollar de acuerdo al entorno o los requerimientos locales, ya que la cultura se adapta pese a que ciertos rasgos culturales no lo hagan (Chakraborty, Thompson, \& Yehoue, 2016; Walter \& Block, 2016).

El emprendimiento suele estar espacialmente agrupado en determinados sectores que, en ocasiones, se alimentan o benefician mutuamente; situación que persiste muchos años y llega a limitar el crecimiento de nuevas compañías. Sin embargo, es interesante analizar si estas diferencias presentes en el mercado se reflejan en las diferencias en el espíritu emprendedor, y en este caso sería la cultura empresarial la que daría inicio a este fenómeno. Siendo este elemento adquirible, las personas mantienen sus probabilidades de éxito (Colino, Benito-Osorio, \& Rueda-Armengot, 2014; Stuetzer \& Obschonka, 2016). Un actor clave para esto es la universidad o centro de formación que se encaje en el área empresarial con pertinencia en las carreras que oferte.

El estudio, se centra en la oferta de la Unidad Académica de Ciencias Empresariales (UACE) de la Universidad Técnica de Machala (UTMACH), en particular, de sus egresados para definir el perfil de cada uno de los grupos que se identificará más adelante. Así, se parte de la necesidad de determinar cuáles serían las habilidades emprendedoras más destacables de los estudiantes egresados de las diferentes carreras de la UACE y cuáles requieren ser reforzadas o entrenadas. Estas destrezas son las que perfilan la cultura emprendedora de los individuos analizados, a la vez, podemos estimar si sus características les permiten desarrollar una iniciativa sustentable de negocio (liyigün, 2015; Swanson \& DeVereaux, 2017).

El objetivo del presente estudio, es analizar la cultura emprendedora que se ha desarrollado en los egresados de las carreras ofertadas por la Unidad Académica de Ciencias Empresariales de la Universidad Técnica de Machala y la sustentabilidad de la misma, para determinar los hábitos más destacables en este grupo y aquellos puntos que requieren se reforzados.

\section{Marco teórico}

El emprendimiento o la capacidad de emprender puede entenderse como un conjunto de costumbres y comportamientos que caracterizan a una persona. Sea que se derive su origen del francés entrepreneur, que significa "contratista o empresario", siendo una persona que bajo su propio riesgo implementa un negocio o empresa; o bien sea un enlace de los vocablos en latín in-prehendere, que 
significa "dentro de la captura", para referirse a la acción de lograr algo que requiere un esfuerzo considerable. Ambos orígenes, o incluso otros más que lo definen como pionero, van por una misma senda que se refiere a invertir un esfuerzo por lograr algo pese a la presencia de riesgos o posibilidad de fracaso (Duman, Bedük, Köylüoğlu, \& Ay, 2015; Swanson \& DeVereaux, 2017). Según David McClelland (1961), Psicólogo, ex Profesor de la Universidad de Harvard en su obra The Achieving Society, analiza los hábitos y maneras de actuar que caracterizan a un emprendedor, las que suelen visualizarse desde tres ángulos según las necesidades que tenga, bien sean de logro, planificación o poder. (Fernández-Salinero \& de la Riva, 2014).

En las necesidades de logro se incluyen los hábitos de búsqueda de oportunidades de negocio y tomar iniciativa en sus acciones, ser persistente en sus objetivos, ser fiel al cumplimiento de un servicio o contrato de trabajo, la exigencia de eficiencia y calidad en las labores que encomienda y realiza, además de tener la capacidad de correr riesgos analizados o calculados. Dentro de las necesidades de planificación se enmarcan las costumbres de fijarse metas medibles y alcanzables dentro de lapso de tiempo, conseguir información relativa al campo que se desea desarrollar o innovar, y la planificación y seguimiento sistemático de las acciones que incluyan un proyecto de negocio propuesto o en marcha. Por último, para las necesidades de poder se consideran hábitos como la capacidad de persuasión, creación de redes de apoyo, y poseer autoconfianza e independencia en las decisiones que toma el negociante (Duman et al., 2015; Sepúlveda R. \& Reina G., 2016).

La cultura emprendedora se refiere a un conjunto específico de conocimientos y habilidades con características especiales que tienen algunos profesionales cuando desarrollan su trabajo, y se también refleja en su comportamiento social. Aquellos empresarios que poseen esta cultura llegan a crear en sus compañías un entorno muy dinámico, agradable y creativo. Ahora bien, a pesar de que parezca una cualidad nata, las personas pueden adquirirla a través del aprendizaje o formación profesional. Sin embargo, aprender la cultura emprendedora no es el objetivo final, porque ésta se adapta incluso cuando algunos rasgos culturales no lo hacen (Chakraborty et al., 2016; Stuetzer \& Obschonka, 2016).

El emprendimiento sustentable implica aquellas innovaciones que se perfilan como oportunidades de negocio, o mejoras en la producción, que beneficien a su entorno minimizando el impacto ambiental que pueda provocar su implementación. Este término incluye a los llamados empresarios sostenibles, o sea, los que facilitan de manera efectiva las demandas latentes de desarrollo sostenible. También se indica que el emprendimiento sustentable fomenta la adaptación de modelos de negocio que sustentan y enaltecen las tradiciones y creencias de una comunidad y los mantienen en el tiempo (Borges de Carvalho, Salete Batista Freitag, Camargo Filho, \& Borges, 2013; Kibler, Fink, Lang, \& Muñoz, 2015; Lans, Blok, \& Wesselink, 2014; Swanson \& DeVereaux, 2017). 


\section{MÉTODO}

$\mathrm{E}$ sta investigación el análisis de la información fue concebida con un enfoque mixto en la escala de medición y resultado de cada perfil analizado. La población estuvo integrada por todos los egresados mencionados y los test se aplicaron dentro de las instalaciones de la UTMACH. Para una población total aproximada de 2100 estudiantes de la UACE que incluye cerca de 850 egresados, la muestra seleccionada fue representativa de la población total distribuida de forma aleatoria estratificada, con las características que se muestran en la Tabla 1. En total se aplican 804 test, es decir, casi el 95\% de los egresados han sido abordados (UTMACH, 2017).

El test aplicado constó de 55 ítems con cuestiones relativas a los hábitos de los emprendedores. Elementos que permiten identificar las denominadas características emprendedoras personales (CEP) en función de tres tipos de necesidades que un profesional tiene, con un total de 10 hábitos enunciados. Las respuestas se abordan con una escala de Likert de 5 niveles de frecuencia, siendo nunca (1) el más bajo y siempre (5) el más alto.

Tabla 1. Distribución de la muestra seleccionada

\begin{tabular}{lrrrr}
\hline CARRERA & Diurna & Vespertina & Nocturna & Subtotal \\
\hline Administración de Empresas & 80 & 40 & 80 & 200 \\
Contabilidad y Auditoría & 80 & 40 & 84 & 204 \\
Comercio Internacional & 80 & 20 & 50 & 150 \\
Ingeniería en Marketing & 80 & 30 & 60 & 170 \\
Administración de Hotelería y Turismo & 40 & No aplica & No aplica & 40 \\
Economía & 40 & No aplica & No aplica & 40 \\
Subtotal & 400 & 130 & 274 & 804 \\
\hline
\end{tabular}

Fuente: (UTMACH, 2017)

Los contenidos del mencionado test propuesto por David McClelland en 1961, ha sido adoptado por la United Nations Conference on Trade and Development (UNCTAD) y ha sido ajustado a muchos estudios recientes, como el de Duman, Bedük, Köylüoğlu, \& Ay (2015) en un caso de estudio en Konya, Turquía; y la ponderación de los resultados se hizo siguiendo los mismos lineamientos que utiliza la Harvard Business School y la Fundación Empretec en su Taller de Desarrollo del Comportamiento Emprendedor (CEFE International, 2017; Duman et al., 2015; EMPRETEC, 2017).

Entonces, se aplicó un diseño de investigación no experimental de tipo transeccional descriptivo y correlacional. Esto último, dado que se estimó la significancia de la relación entre algunos de los indicadores (hábitos) analizados. Después de obtener la información, la escala sugerida se probará con los resultados mediante una prueba de fiabilidad con Alfa de Cronbach, y la distribución de éstos con una prueba de normalidad con coeficiente de Shapiro-Wilk, dado que cada muestra no supera los 100 
elementos (Lind \& Marchal, 2012). Para obtener el factor de corrección individual (FCI) se partió del factor de corrección general (FCG), y se guío por lo siguiente:

- $\quad F C G=19$ ó menor, $F C I=0$

- $\quad F C G=20$ ó $21, F C I=3$

- $\quad F C G=22$ ó $23, F C I=5$

- $\quad F C G=24$ ó $25, F C I=7$

La puntuación final se ubica entre 0 a 25 puntos para cada característica, luego este valor puede fácilmente transformarse a otras escalas decimales o porcentuales para una mejor interpretación. Asimismo, una representación gráfica es de utilidad para presentar los resultados individuales o promediados para grupos específicos. En este caso, se presentan por carrera y sección (diurna, vespertina, nocturna); después, estos se contrastan entre sí como datos de grupo y también se muestra un promedio general (CEFE International, 2017). Las calificaciones que se obtuvieron luego de la aplicación de los test para cada característica emprendedora personal (denominados como hábitos emprendedores) se cuantificó en porcentaje de acuerdo a lo detallado en la Tabla 2.

Tabla 2. Cualificación de los puntajes obtenidos en los test aplicados

\begin{tabular}{ccl}
\hline Rango de calificación & Porcentaje relativo & \multicolumn{1}{c}{ Cualificación } \\
\hline $22.51-25.00$ & $91-100 \%$ & Excelente \\
$20.01-22.50$ & $81-90 \%$ & Muy bueno \\
$17.51-20.00$ & $71-80 \%$ & Bueno \\
$15.01-17.50$ & $61-70 \%$ & Regular \\
$00.00-15.00$ & $0-60 \%$ & Deficiente \\
\hline
\end{tabular}

Asimismo, éstos se cualificarán, para su discusión, según lo propuesto en el mismo cuadro. Por la naturaleza cuantitativa de los resultados (puntuaciones), la correlación entre los indicadores se hará usando el coeficiente de Pearson.

\section{RESULTADOS}

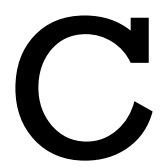

on un alfa de Cronbach del $88.3 \%$ para la escala usada para los ítems aplicados en la encuesta, se evidenció una alta confiabilidad de los resultados obtenidos. Las pruebas de normalidad revelaron, de forma general, que los datos recogidos no se encuentran distribuidos normalmente; esto se confirmó puesto que sólo en 11 de los 60 conjuntos analizados (6 carreras por 10 hábitos) la significancia supera el 5\%, condición que confirmó la probable 
normalidad de los registros. Estos conjuntos son 7 de la Carrera Administración de Hotelería y Turismo, y 4 de la Carrera de Economía. El primero de estos dos grupos podría ser analizado por separado en caso que sus resultados así lo ameriten. No obstante, la calificación obtenida por esta carrera está por debajo de varias otras relativas.

Los egresados que mejor cultura emprendedora perfilan fueron los de las Carreras de Comercio Internacional y los de Ingeniería en Marketing. En la Tabla 3 se presentan algunos valores estadísticos descriptivos de las puntuaciones obtenidas por los egresados de UACE de forma conjunta. Se aprecia que, con una desviación estándar similar en todos los casos, el hábito emprendedor que más sobresale es el de fijarse metas claras a corto y largo plazo (puntuación promedio 17.04). El aspecto más débil tiene que ver con la capacidad de correr riesgos calculados puntuación promedio 16.37). Estos resultados dejan entrever que la diferencia entre calificaciones es relativamente baja.

Tabla 3. Estadísticos descriptivos de los resultados generales

\begin{tabular}{lccccr}
\hline Hábitos emprendedores & N & Media & $\begin{array}{c}\text { Desviación } \\
\text { estándar }\end{array}$ & Mínimo & Máximo \\
\hline Buscar oportunidades y tener iniciativa & 804 & 16,91 & 2,358 & 7 & 24 \\
Ser persistente & & & & & \\
Ser fiel a cumplimiento del contrato de trabajo & 804 & 16,93 & 2,445 & 6 & 24 \\
& & 16,92 & 2,441 & 8 & 24 \\
Exigir eficiencia y calidad & 804 & 16,77 & 2,404 & 10 & 25 \\
Correr riesgos & 804 & 16,37 & 2,561 & 7 & 23 \\
Fijar metas & 804 & 17,04 & 2,652 & 8 & 25 \\
Conseguir información & 804 & 16,71 & 2,332 & 9 & 24 \\
Planificar y hacer seguimiento sistemático & 804 & 16,61 & 2,505 & 6 & 23 \\
Ser persuasivo y crear redes de apoyo & 804 & 16,54 & 2,522 & 8 & 24 \\
Tener autoconfianza & 804 & 16,88 & 2,575 & 8 & 23 \\
\hline
\end{tabular}

En el Gráfico 1 acerca de la representación del perfil general de egresados de la UACE. Para determinar si hay efectos por categorías de carreras o de sección, es decir, si en alguna de estas se marcan mejores calificaciones, siendo los registros de partida no normales, aplicamos una prueba de Kruscal-Wallis 


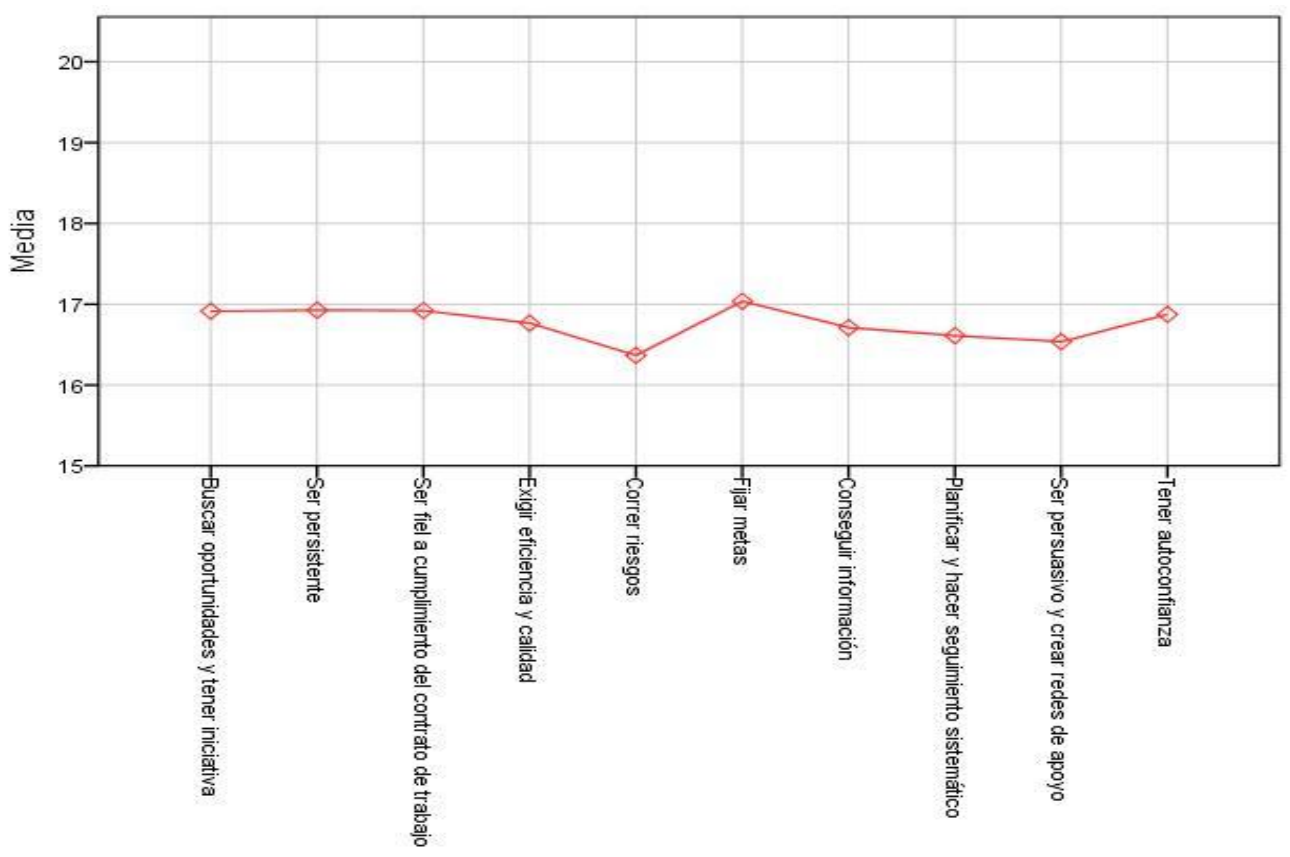

Gráfico 1. Perfil general de egresados de la Unidad Académica de Ciencias Empresariales.

Tal como lo muestra la Tabla 4, todas las carreras tienen resultados estadísticamente distintos, siendo la Carrera de Comercio Internacional la más sobresaliente respecto al resto. Esto se puede constatar con los valores de los rangos o directamente con las calificaciones promediadas. Los hábitos más destacables son la autoconfianza, buscar oportunidades de negocio y tener iniciativa, y obtener información para emprender algo sustentable.

Tabla 4. Pruebas de Kruskal-Wallis para contraste de hipótesis (categorías de carreras)

\begin{tabular}{|c|c|c|c|c|}
\hline Hábitos emprendedores & Chi cuadrado & $\begin{array}{l}\text { Grados de } \\
\text { libertad }\end{array}$ & $\begin{array}{l}\text { Significación } \\
\text { asintótica }\end{array}$ & Decisión \\
\hline $\begin{array}{l}\text { Buscar oportunidades y tener } \\
\text { iniciativa }\end{array}$ & 53,718 & 5 & 0,000 & Rechazar hipótesis nula \\
\hline Ser persistente & 45,505 & 5 & 0,000 & Rechazar hipótesis nula \\
\hline Exigir eficiencia y calidad & 17,231 & 5 & 0,004 & Rechazar hipótesis nula \\
\hline Correr riesgos & 26,654 & 5 & 0,000 & Rechazar hipótesis nula \\
\hline
\end{tabular}




\begin{tabular}{|c|c|c|c|c|}
\hline Hábitos emprendedores & Chi cuadrado & $\begin{array}{l}\text { Grados de } \\
\text { libertad }\end{array}$ & $\begin{array}{l}\text { Significación } \\
\text { asintótica }\end{array}$ & Decisión \\
\hline Fijar metas & 26,624 & 5 & 0,000 & Rechazar hipótesis nula \\
\hline Conseguir información & 57,256 & 5 & 0,000 & Rechazar hipótesis nula \\
\hline $\begin{array}{l}\text { Planificar y hacer seguimiento } \\
\text { sistemático }\end{array}$ & 41,021 & 5 & 0,000 & Rechazar hipótesis nula \\
\hline $\begin{array}{l}\text { Ser persuasivo y crear redes de } \\
\text { apoyo }\end{array}$ & 21,723 & 5 & 0,001 & Rechazar hipótesis nula \\
\hline Tener autoconfianza & 88,442 & 5 & 0,000 & Rechazar hipótesis nula \\
\hline
\end{tabular}

En la misma línea anterior, en la Tabla 5 se muestra que para la categoría secciones sólo hay diferencia significativa (al $1 \%$ de error) en dos de los elementos, destacando en ambos casos la sección vespertina para los hábitos de exigir eficiencia y calidad para nuestros trabajos, y ser persuasivo y crear redes de apoyo.

La validación se hace por medio de herramientas de análisis básico, como lo son las hipótesis estadísticas. Los criterios usados para la interpretación de los resultados, en ambos casos de categorías, son los siguientes:

- $\quad$ Hipótesis nula: No hay diferencia significativa entre los grupos analizados.

- Hipótesis alterna: Existe diferencia significativa entre al menos dos de los grupos. 
Tabla 5. Pruebas de Kruskal-Wallis para contraste de hipótesis (categorías de sección)

\begin{tabular}{|c|c|c|c|c|}
\hline Hábitos & $\begin{array}{c}\text { Chi - } \\
\text { cuadrado }\end{array}$ & $\begin{array}{l}\text { Grados de } \\
\text { libertad }\end{array}$ & $\begin{array}{l}\text { Significación } \\
\text { asintótica }\end{array}$ & Decisión \\
\hline Buscar oportunidades y tener iniciativa & 0,068 & 2 & 0,966 & $\begin{array}{c}\text { Aceptar } \\
\text { hipótesis } \\
\text { nula }\end{array}$ \\
\hline Ser persistente & 6,068 & 2 & 0,048 & $\begin{array}{c}\text { Aceptar } \\
\text { hipótesis } \\
\text { nula }\end{array}$ \\
\hline Ser fiel a cumplimiento del contrato de trabajo & 2,484 & 2 & 0,289 & $\begin{array}{c}\text { Aceptar } \\
\text { hipótesis } \\
\text { nula }\end{array}$ \\
\hline Exigir eficiencia y calidad & 9,458 & 2 & 0,009 & $\begin{array}{c}\text { Rechazar } \\
\text { hipótesis } \\
\text { nula }\end{array}$ \\
\hline Correr riesgos & 3,760 & 2 & 0,153 & $\begin{array}{c}\text { Aceptar } \\
\text { hipótesis } \\
\text { nula }\end{array}$ \\
\hline Fijar metas & 0,084 & 2 & 0,959 & $\begin{array}{c}\text { Aceptar } \\
\text { hipótesis } \\
\text { nula }\end{array}$ \\
\hline Conseguir información & 7,724 & 2 & 0,021 & $\begin{array}{c}\text { Aceptar } \\
\text { hipótesis } \\
\text { nula }\end{array}$ \\
\hline Planificar y hacer seguimiento sistemático & 1,590 & 2 & 0,452 & $\begin{array}{c}\text { Aceptar } \\
\text { hipótesis } \\
\text { nula }\end{array}$ \\
\hline Ser persuasivo y crear redes de apoyo & 26,988 & 2 & 0,000 & $\begin{array}{c}\text { Rechazar } \\
\text { hipótesis } \\
\text { nula }\end{array}$ \\
\hline Tener autoconfianza & 3,221 & 2 & 0,200 & $\begin{array}{c}\text { Aceptar } \\
\text { hipótesis } \\
\text { nula }\end{array}$ \\
\hline
\end{tabular}

A continuación, (Tabla 6) se presentan las correlaciones entre indicadores o hábitos, estimadas con coeficiente de Pearson. Sólo en una de las 25 interacciones la significancia es apenas mayor al 1\%. Esto denota que las interacciones difícilmente se las pueden atribuir a la propia distribución aleatoria de la información receptada. Todas las habilidades de los emprendedores se relacionan; cuando una sube las otras también, y si hay deficiencias esto se va a reflejar en una disminución de todas las calificaciones. 
Así, el nexo más fuerte se presenta en la fijación de metas y el cumplimiento cabal de las obligaciones o contratos. Esto induce a pensar que las necesidades de logro están fuertemente ligadas a las de planificación.

El nexo más débil estuvo entre los hábitos de persistencia y la persuasión y creación de redes de apoyo. Su validez se enmarca para un nivel de significancia muy inferior al 5\%, siendo apenas superior al $1.1 \%$ en realidad. Esta última aseveración no invalida o se contrapone a las últimas, pero sí manifiesta una leve deficiencia en la incidencia de las necesidades de logro y las de poder. Los estudiantes egresados de la Unidad Académica de Ciencias Empresariales de la Universidad Técnica de Machala han de buscar programas, seminarios o talleres que les permitan fortalecer sus hábitos, desarrollando una cultura emprendedora que sea sustentable con el medio ambiente y el entorno local con miras a su desarrollo.

En este sentido, probablemente, la educación que se imparte en función de la carrera que elige el estudiante este influyendo en su cultura emprendedora, dado que todos los perfiles tienen diferencias significativas. La sustentabilidad de sus ideas dependería, por tanto, de la inducción recibida a través de proyectos e investigaciones que desarrollen durante la formación profesional, en este caso, en la Universidad Técnica de Machala.

Tabla 6. Análisis de correlaciones entre indicadores (hábitos emprendedores)

\begin{tabular}{|c|c|c|c|c|c|c|}
\hline Hábitos & Estadístico & 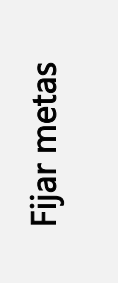 & 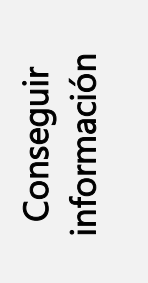 & 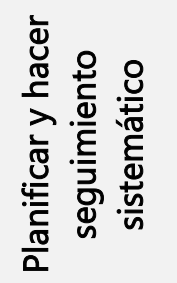 & 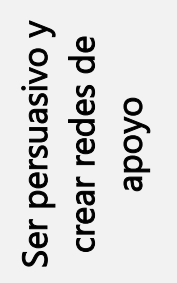 & 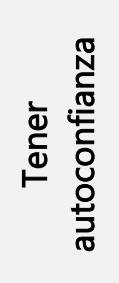 \\
\hline \multirow{3}{*}{$\begin{array}{l}\text { Buscar oportunidades y tener } \\
\text { iniciativa }\end{array}$} & $\begin{array}{l}\text { Correlación } \\
\text { de Pearson }\end{array}$ &, $185^{* *}$ &, $123^{* *}$ &, $201^{* \star}$ &, $194^{* *}$ & $214^{* \star}$ \\
\hline & Sig. (bilateral) & ,000 & ,000 &, 000 &, 000 & ,000 \\
\hline & $\mathrm{N}$ & 804 & 804 & 804 & 804 & 804 \\
\hline \multirow{3}{*}{ Ser persistente } & $\begin{array}{l}\text { Correlación } \\
\text { de Pearson }\end{array}$ & $228^{* *}$ &, $175^{* *}$ &, $178^{* *}$ &, $090^{*}$ &, $204^{\star \star}$ \\
\hline & Sig. (bilateral) &, 000 & ,000 & ,000 & 011 & ,000 \\
\hline & $\mathrm{N}$ & 804 & 804 & 804 & 804 & 804 \\
\hline \multirow{3}{*}{$\begin{array}{l}\text { Ser fiel a cumplimiento del contrato } \\
\text { de trabajo }\end{array}$} & $\begin{array}{l}\text { Correlación } \\
\text { de Pearson }\end{array}$ & $295^{* *}$ &, $193^{* \star}$ &, $179^{* \star}$ &, $123^{* *}$ &, $130^{* \star}$ \\
\hline & Sig. (bilateral) &, 000 &, 000 &, 000 &, 000 &, 000 \\
\hline & $\mathrm{N}$ & 804 & 804 & 804 & 804 & 804 \\
\hline
\end{tabular}




\begin{tabular}{|c|c|c|c|c|c|c|}
\hline Hábitos & Estadístico & 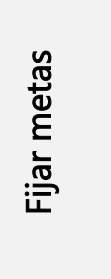 & 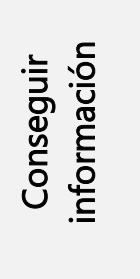 & 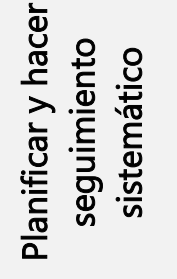 & 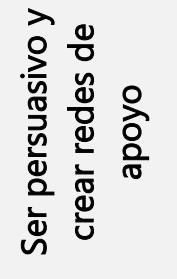 & 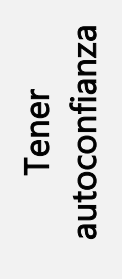 \\
\hline \multirow{3}{*}{ Exigir eficiencia y calidad } & $\begin{array}{l}\text { Correlación } \\
\text { de Pearson }\end{array}$ &, $159^{\star *}$ &, $137^{* *}$ &, $137^{* *}$ &, $168^{* *}$ &, $117^{* \star}$ \\
\hline & Sig. (bilateral) &, 000 &, 000 &, 000 &, 000 &, 001 \\
\hline & $\mathrm{N}$ & 804 & 804 & 804 & 804 & 804 \\
\hline \multirow{3}{*}{ Correr riesgos } & $\begin{array}{l}\text { Correlación } \\
\text { de Pearson }\end{array}$ &, $263^{* *}$ &, $175^{\star *}$ &, $133^{* *}$ &, $235^{* *}$ &, $234^{* \star}$ \\
\hline & Sig. (bilateral) &, 000 &, 000 &, 000 &, 000 &, 000 \\
\hline & $\mathrm{N}$ & 804 & 804 & 804 & 804 & 804 \\
\hline \multicolumn{7}{|c|}{ **. La correlación es significativa en el nivel 0,01 (bilateral). } \\
\hline
\end{tabular}

\section{CONCLUSIONES}

$\mathrm{L}$ a estadística reveló una significancia representativa (al 99\% de nivel de confianza) en la diferencia entre categorías de carreras, el grupo que sobresalió fue el de egresados de la carrera de Comercio Internacional. La fortaleza de estos estudiantes se centró en sus necesidades de logro, en especial en los hábitos de buscar oportunidades de emprender negocios nuevos, sustentables ecológicos y pertinentes a las demandas locales, y tener la iniciativa para cubrir alguna demanda insatisfecha en productos o servicios de posibles clientes (esto también es destacable en los estudiantes de Ingeniería en Marketing), aparte de exigir eficiencia y calidad para garantizar los resultados de un trabajo. También destacó el hecho de tener autoconfianza e independencia en sus decisiones, asumiendo tanto sus éxitos como sus fracasos, este elemento se enfrasca en las necesidades de poder.

Las debilidades de este grupo de estudiantes, curiosamente se evidenció en esta última característica, al faltarles habilidades de persuasión y para formar redes de apoyo que les faciliten vincularse con otras personas o empresas para potenciar sus posibilidades de triunfo dentro de un negocio. Cabe mencionar, que ellos además requieren mejorar en cuanto a planificación, en específico, fijarse metas $u$ objetivos que tengan relevancia dentro de su formación personal y profesional. En adición vale destacar, que los estudiantes de la sección vespertina de esta carrera mostraron un buen perfil (el mejor en relación a las otras secciones) en todas las características de comportamiento emprendedor. Por tanto, valdría considerar a este grupo para inducir a que desarrollen proyectos con el uso de tecnologías más limpias, que tributen al desarrollo local sustentable de sus comunidades. 
Otro grupo de egresados que sobresalen del promedio habitual son de la carrera de Ingeniería en Marketing, que además de la búsqueda continua de oportunidades de negocio e iniciativa, reflejaron un nivel relevante en cuanto al cumplimiento de las tareas a ellos encomendadas o los contratos que suscriben, hábitos que se caracterizan como necesidades de logro dentro de su desempeño. No obstante, en esta misma característica los limita la capacidad de correr riesgos calculados o analizados adecuadamente. A esto último, se le suma la baja persuasión que pueden crear y deficiente manejo o formación de probables redes de apoyo, incluyendo autoconfianza relativamente baja y tendencia a perder independencia en sus decisiones o a no reconocer sus aciertos y errores.

Además, cabe mencionar a los egresados de la carrera de Administración de Empresas, quienes resaltaron cuando se requiere a fijar metas claras a corto y largo plazo, medibles y dignas de reconocimiento. Este hábito refuerza la característica de necesidad de planificación. En contraparte, las necesidades de logro para este grupo de estudiantes son deficiente hasta cierto punto, en especial cuando buscan nuevas oportunidades de emprendimientos, tienen una iniciativa regular e inseguridad para arriesgarse en un negocio que previamente haya evaluado. La característica referida al poder es otra que necesitan mejorar este grupo de egresados, que se han de enfocar en su capacidad de persuasión y habilidad para crear grupos o alianzas de apoyo a sus ideas de negocio.

Por otro lado, el grupo que más atención requiere en materia de capacitación e inducción de una cultura emprendedora fue el compuesto por los estudiantes de la carrera de Contabilidad y Auditoría. Sus necesidades de logro y de planificación son apenas regulares en cuanto a la búsqueda de oportunidades e iniciativa propia, la exigencia de eficiencia y calidad para sus requerimientos, y la fijación de metas claras. Sus más grandes debilidades se centran en sus necesidades de poder, ya que son poco persuasivos y deficientes para crear redes de apoyo, con baja autoconfianza e independencia en sus decisiones. Además, la peor calificación que obtuvo este grupo fue en el indicador planificación y seguimiento sistemático, relativo a las necesidades de planificación que tienen los emprendedores.

Finalmente, al referirse de hábitos neutros o de relevancia poco significativa, estas serían la persistencia y la búsqueda de información, la primera de ellas relativa a la característica de comportamiento en necesidad de logro y la segunda tiene que ver con la de planificación. La sustentabilidad de los emprendimientos que puedan generar los egresados de la UACE de la UTMACH, es tomada como parte de la propia iniciativa de este grupo, y que ha sido inducida dentro de su formación profesional mediante la participación en proyectos de vinculación con la colectividad. Es así que las nuevas ideas de negocio que ellos propongan han de ser sustentables desde el plano ecológico y propiciar el desarrollo local.

Esta investigación puede servir como base para proponer programas o talleres de formación y mejoramiento de la cultura emprendedora y sustentable de los estudiantes o de profesionales incluso, luego de haber analizado sus características de comportamiento, para así personalizar las capacitaciones, con conciencia ecológica y solidaria, y optimizar los resultados de las mismas. 


\section{REFERENCIAS}

Borges de Carvalho, J.; Salete, M.; Camargo, A., y Borges, C. (2013). El aprendizaje empresarial en los emprendimientos sustentables del sector turistico del cerrado brasileno. Estudios y Perspectivas en Turismo

CEFE International. (2017). CEFE International. Retrieved from http://cefe.net/

Chakraborty, S.; Thompson, J. C., y Yehoue, E. B. (2016). The culture of entrepreneurship. Journal of Economic Theory. pp. 288-317

Colino, A.; Osorio, D., y Rueda, C. (2014). Entrepreneurship culture, total factor productivity growth and technical progress: Patterns of convergence towards the technological frontier. Technological Forecasting and Social Change, pp.349-359

Duman, L.; Bedük, A.; Köylüoğlu, A. S., y Ay, K. (2015). Entrepreneurship Culture at SMEs: A Case Study in Konya. Procedia - Social and Behavioral Sciences, pp. 207, 492-501

EMPRETEC. (2017). Fundación EMPRETEC Argentina

Fernández, C., y de la Riva, B. (2014). Entrepreneurial Mentality and Culture of Entrepreneurship. Procedia - Social and Behavioral Sciences, pp. 139, 137-143

lyigün, N. Ö. (2015). What could Entrepreneurship do for Sustainable Development A Corporate Social Responsibility-Based Approach. Procedia - Social and Behavioral Sciences, pp. 195, 12261231
Kibler, E.; Fink, M.; Lang, R., y Muñoz, P. (2015). Place attachment and social legitimacy: Revisiting the sustainable entrepreneurship journey. Journal of Business Venturing Insights, pp. 3, 24-29

Lans, T., Blok, V., y Wesselink, R. (2014). Learning apart and together: towards an integrated competence framework for sustainable entrepreneurship in higher education. Journal of Cleaner Production, pp. 62, 37-47

Lind, D., \& Marchal, W. (2012). Estadistica aplicada a los negocios y la economía. (Vol. 15)

Sepúlveda R., C., y Reina G., W. (2016). Sostenibilidad de los emprendimientos: Un análisis de los factores determinantes. Revista Venezolana de Gerencia ( $R V G$, pp. 73, 33-50

Stuetzer, M., y Obschonka, M. (2016). Industry structure, entrepreneurship, and culture: An empirical analysis using historical coalfields. European Economic Review

Swanson, K. K., y DeVereaux, C. (2017). A theoretical framework for sustaining culture: Culturally sustainable entrepreneurship. Annals of Tourism Research, pp. 62, 78-88

UTMACH. (2017). Universidad Técnica de Machala

Walter, S. G., y Block, J. H. (2016). Outcomes of entrepreneurship education: An institutional perspective. Journal of Business Venturing, pp. $31(2), 216-233$ 